\title{
IMF variations in unresolved stellar populations: Challenges
}

\author{
Ignacio Ferreras ${ }^{1}$, Francesco La Barbera ${ }^{2}$ and Alexandre Vazdekis ${ }^{3}$ \\ ${ }^{1}$ MSSL, University College London, Holmbury St Mary, Dorking, Surrey, RH5 6NT, UK \\ 2 INAF-Osservatorio Astronomico di Capodimonte, sal. Moiariello 16, Napoli I-80131, Italy \\ ${ }^{3}$ IAC and Universidad de La Laguna, C/ Vía Láctea s/n, E-38205 La Laguna, Tenerife, Spain \\ email: i.ferreras@ucl.ac.uk
}

\begin{abstract}
This talk focuses on the challenges facing the recent discovery of variations of the stellar initial mass function in massive early-type galaxies, with special emphasis on the constraints via gravity-sensitive spectral features.
\end{abstract}

Keywords. stars: luminosity function, mass function, galaxies: elliptical and lenticular, cD

\section{Challenges facing studies of IMF variations}

The stellar initial mass function (IMF) is one of the pillars of any stellar population synthesis models. It allows us to create synthetic spectra of galaxies from which one can derive fundamental parameters such as the star formation rate or the stellar mass. The IMF has been commonly treated as a "fixed" distribution, anchored by the observations of resolved populations in our galaxy. Over the past few years, several papers revealed a significant trend of IMF-sensitive spectral indices in early-type galaxies (ETGs), such that, at the high-mass end, the central regions of ETGs feature an excess of low-mass stars with respect to a Milky Way standard IMF (van Dokkum \& Conroy 2010). Independently, studies based on dynamical modelling or gravitational lensing give similar trends, with a heavier M/L in the most massive ETGs (Cappellari et al 2012,Posacki et al. 2015). This invited talk presented an overview of the challenges facing these type of analyses.

\subsection{Challenge \# 1: Observational pitfalls}

There are two main aspects to this challenge. Many of the gravity-sensitive spectral features live in the red/NIR part of the spectrum, with the consequent complication from airglow emission and telluric absorption. A careful selection of the redshift of the targets can help alleviate this issue, "moving" the features into the cleanest areas of the red/NIR spectral range. In addition, the variations from a change in the IMF are small, at the few percent level with respect to the continuum. Therefore, the observations require especially large SNR, usually of order a few hundred per Angstrom.

\subsection{Challenge \# 2: Interpretation of the line strengths}

The line strengths of an unresolved population are the result of a superposition of a large number of stars over a wide range of ages and chemical composition. Moreover, these lines are heavily blended due to the high velocity dispersion of the stars. This problem is especially acute in massive ETGs - where the IMF departure from a Milky Way standard is largest. The outcome of this blending is a large degeneracy between IMF variations and other population parameters, such as the age, the chemical composition (as an overall metallicity, a more detailed $[\alpha / \mathrm{Fe}]$, or even for individual element abundances), or 
isochrone-related parameters (e.g. temperature scale). The way to mitigate these degeneracies involves an extended study of many spectral features sensitive to these factors in different degrees (see, e.g., La Barbera et al. 2013).

\subsection{Challenge \# 3: Driving mechanisms of IMF variations}

What is the major driver of such a change? Here, different authors suggest different options, such as $[\mathrm{Mg} / \mathrm{Fe}]$ (Conroy \& van Dokkum 2012), velocity dispersion (Ferreras et al. 2013, La Barbera et al. 2013), concentration (Spiniello et al. 2015) or metallicity (MartínNavarro et al. 2015b). Ultimately, this question requires both a detailed understanding of the underlying degeneracies (Smith 2014; La Barbera et al. 2015a) and resolved spectroscopic observations that reveal variations within galaxies (Martín-Navarro et al. 2015a,b).

\subsection{Challenge \# 4: Stellar $M / L$ constraints}

The three independent probes of the IMF of the passive populations in ETGs (spectral features, dynamical modelling, gravitational lensing) can be combined to impose further constraints on the functional form of the IMF. The latter two methods are sensitive to the stellar M/L, whereas spectroscopic measurements constrain the ratio of giant-to-dwarf stars. Hence, combining these methods one can distinguish between different scenarios that give rise to a bottom-heavy IMF. Recent results based on lensing have challenged the consistency of this analysis, with a few massive ETGs having relatively low stellar M/L (Smith \& Lucey 2013). More work is undoubtedly needed to constrain the functional form of the IMF (e.g. La Barbera et al. 2015b rule out a single power law IMF).

\subsection{Challenge \# 5: Chemical enrichment}

Finally, if we give in to the mounting evidence and accept a bottom-heavy IMF in massive ETGs, we need to revisit galactic chemical enrichment. Given the early, intense and short-lived star formation history of massive ETGs, such an IMF will lock too many stars at very low metallicities, in stark disagreement with the observations. The only way to reconcile all these observables is to assume that, further to a variation among ETGs, there needs to be a time dependence, where the bottom-heavy phase takes place during the later stages of star formation, after which the metallicity of the ISM is high enough (Weidner et al. 2013). This scenario is compatible with the theoretical ideas of an enhanced fragmentation in highly turbulent star forming regions (Chabrier et al. 2014).

\section{References}

Cappellari, M., et al. 2012, Nature, 484, 485

Chabrier, G., Hennebelle, P., \& Charlot, S. 2014, ApJ, 796, 75

Conroy, C. \& van Dokkum, P. 2012, ApJ, 760, 71

Ferreras, I., et al. 2013, MNRAS, 429, L15

La Barbera, F., et al. 2013, MNRAS, 433, 3017

La Barbera, F., et al. 2015a, MNRAS, 449, L137

La Barbera, F., et al. 2015b, arXiv, 1509.08250

Martín-Navarro, I., et al. 2015a, MNRAS, 447, 1033

Martín-Navarro, I., et al. 2015b, ApJ, 806, L31

Posacki, S., et al., 2015, MNRAS, 446, 493

Smith, R. J. \& Lucey, J. R. 2013, MNRAS, 434, 1964

Smith, R. J. 2014, MNRAS, 443, L69

Spiniello, C., et al. 2015, MNRAS, 452, L21

van Dokkum, P. \& Conroy, C. 2010, Nature, 468, 940

Weidner, C., et al. 2013, MNRAS, 435, 2274 